\title{
Three dimensional wakes of freely falling planar polygons
}

\author{
Luis Blay Esteban $^{1}$ [ $\cdot$ John Shrimpton ${ }^{1} \cdot$ Bharathram Ganapathisubramani $^{1}$
}

Received: 25 February 2019 / Revised: 6 June 2019 / Accepted: 6 June 2019 / Published online: 18 June 2019

(c) The Author(s) 2019

\begin{abstract}
The wake characteristics of various thin particles with identical material properties but different frontal geometries (disks, hexagonal plates and square plates) are examined by means of three dimensional measurements of the instantaneous velocity field. The reference particle is a circular disk that lies within the Reynolds number-dimensionless moment of inertia domain $\left(R e-I^{*}\right)$ corresponding to the fluttering regime, as defined by Willmarth et al. (Phys Fluids 7:197-208, 1964). Hexagonal and square plates are manufactured to have the same frontal area and material properties of the reference particle. Three dimensional trajectories obtained from high-speed imaging show that disks preferably adopt a quasi-2D oscillatory descent; i.e. 'planar zig-zag', whereas particles with less circularity adopt three dimensional trajectories more frequently; i.e. 'transitional' and 'spiral' descent. The wake behind free-falling disks is found to be a succession of hairpin vortices shed off at every turning point linked by a pair of counter rotating vortices that grow downstream from the leading edge of the disk. In contrast, square plates describing 'spiral' descent show an almost time-independent wake morphology with large-scale vortex shedding around the entire perimeter of the particle. The large-scale wake structures of hexagonal plates resemble either the disks' or the squares' depending on the falling regime that they adopt. Finally, we compare the dimensionless vorticity distribution in the wake of the particles and found that this also depends on the falling style that the particle adopts during the descent.
\end{abstract}

\section{Graphic abstract}
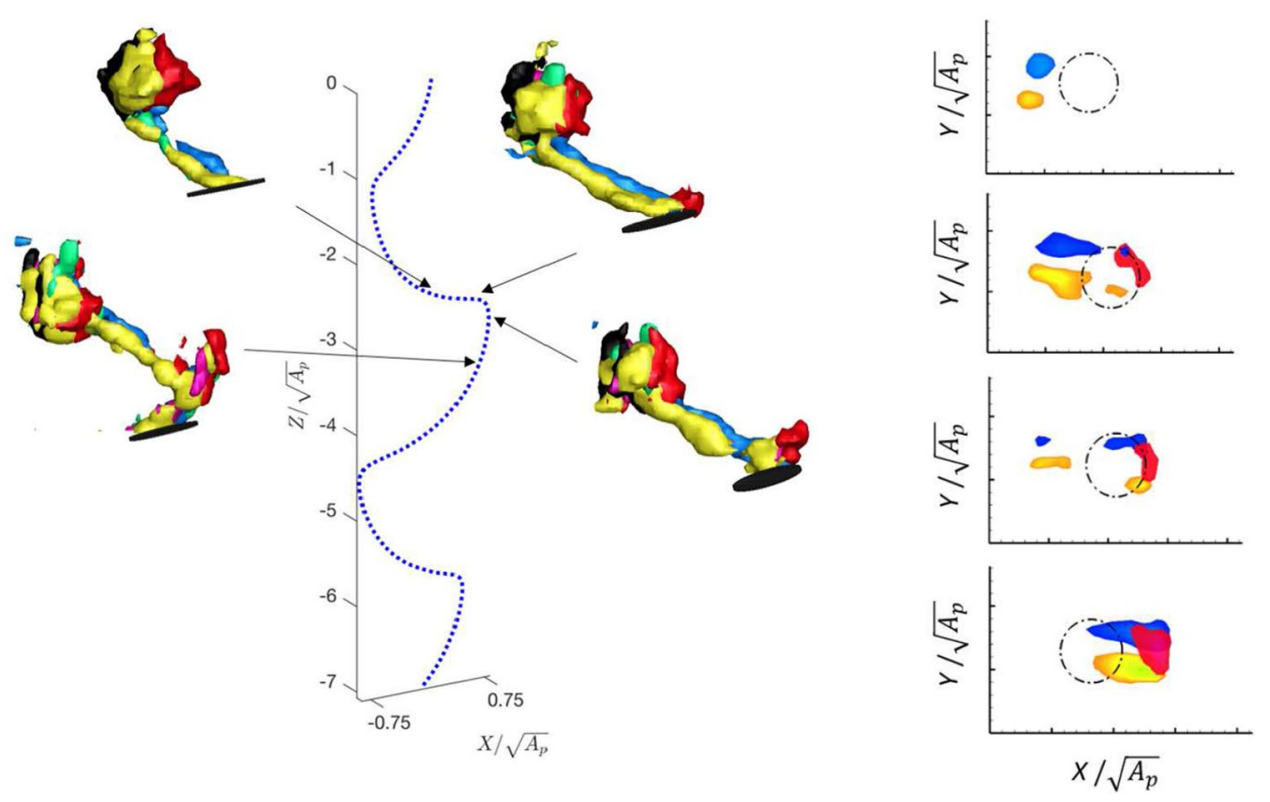

Extended author information available on the last page of the article 


\section{Introduction}

The physical mechanisms involved in the free fall of particles in a viscous media have been investigated in many studies across a broad range of disciplines, from earth science to engineering problems. Examples of these phenomena in natural environments include seed dispersal (Sabban and van Hout 2011), the deposition of volcanic ash (Wilson and Huang 1979), the falling of rain droplets and ice crystals (List and Schemenauer 1971; Jayaweera 1972; Kajikawa 1992), and sedimentation processes as in Feng et al. (1994). Industrial processes include the generation of pollutants by combustion processes, as described in Jones et al. (2014). Fundamental research of free falling particles has focused on wake structures, vortex shedding mechanisms and body-to-fluid interactions with the objective of understanding the characteristics of the paths of the falling objects.

The first phase diagram that characterised the falling regime of disks was defined experimentally by Willmarth et al. (1964). They mapped three different falling regimes; i.e. steady, fluttering and tumbling, and discussed that the phase diagram held for disks as long as the aspect ratio $(\chi)$, defined as $\chi=D / h$ (where $h$ is the thickness of the disk and $D$ the diameter), was small. Thus, they were able to predict the falling style of a given disk once the Reynolds number $(R e)$ and the dimensionless moment of inertia $\left(I^{*}\right)$ were known. The Reynolds number defined as $R e=\left\langle V_{\mathrm{z}}\right\rangle D / v$, where $\left\langle V_{\mathrm{z}}\right\rangle$ is the average settling velocity and $v$ is the kinematic viscosity of the fluid. The dimensionless moment of inertia as $I^{*}=I_{\mathrm{p}} / \rho_{\mathrm{f}} D^{5}$, where $I_{\mathrm{p}}$ is the mass moment of inertia about the diameter of the disk and $\rho_{f}$ is the density of the fluid. Following a similar approach, Smith (1971) constructed the corresponding phase diagram for quasi $2 D$ rectangular plates.

Field et al. (1977) identified a new falling regime that lies in between the fluttering and tumbling regimes. This regime was labelled as 'chaotic' due to the apparent unpredictable particle motion during the descent. This new falling style has been investigated in more detail during the past decades, especially for quasi $2 D$ rectangular plates, as in Belmonte et al. (1998), Andersen et al. (2005a, b).

Fernandes et al. (2007) performed a numerical investigation on the oscillatory motion of axisymmetric bodies in the range of $80<\operatorname{Re}<330$ and $1.5<\chi<20$ and found different body motion and wake characteristics as a function of the body aspect ratio $\chi$. They showed that as $\chi$ increases, the axial symmetry of the wake and the wake oscillations appeared at a smaller critical Reynolds number. They distinguished between thick $(\chi<6)$ and thin $(\chi>6)$ bodies based on the comparison between the shedding frequency of a freely falling disk with a fixed body wake. Also, they showed that the frequency and the amplitude of the oscillations are independent of $\chi$ when they are made non-dimensional with the appropriate length and velocity scale, $D$ and $\sqrt{\left(\rho_{\mathrm{p}} / \rho_{\mathrm{f}}-1\right) g D}$, respectively.

Zhong et al. (2011) focused on the falling style of thin disks with very low dimensionless moment of inertia, corresponding to a region of the phase diagram associated with the fluttering regime. They showed that the descent paths of these particles exhibit a transition from 'planar zig-zag' to 'spiral' motion that is in fact determined by the dimensionless moment of inertia and the Reynolds number. They also carried flow visualization and showed that the change in path style comes with a drastic change in the turbulent structures present in the wake. Lee et al. (2013) extended the work of Zhong et al. (2011) investigating the transition between these two falling modes. They also performed planar Particle Image Velocimetry (PIV) measurements on the particle wake, reaffirming the findings previously observed qualitatively by Zhong et al. (2011). Interestingly, a study focused on the landing position distribution of disks falling under different descent modes (Heisinger et al., 2014) showed that a disk (with a given $R e$ and $I^{*}$ ) can follow both types of motion; i.e. 'planar zig-zag' and 'spiral' at different realizations. This suggests that the falling motion of a given particle is not unique and therefore should be examined through statistical analysis.

Several direct numerical simulations studies, as Auguste et al. (2013) and Chrust et al. (2013), have focused on the descent style of thin disks for small to moderate Reynold numbers $(R e<300)$ and found remarkable differences in the dynamics of disks for different $\chi$ values. However, these modes are only present at moderate Reynolds number and will not be further discussed in this study.

Fewer studies are available for other planar particles than disks and rectangular plates. Early investigations were carried out by List and Schemenauer (1971) on the steady fall of planar irregular particles relative to a reference disk. These included hexagonal plates, broad-branched crystals and stellar crystals. In addition to an increase in drag coefficient with Reynolds number associated with an increase in perimeter (due to viscous effects), they also observed small self-induced oscillations during the fall. These secondary motions were qualitatively smaller for the irregular particles than for the case of the reference disk. Jayaweera (1972) also studied the steady free fall of several planar particles and showed that the terminal velocity of star-shape particles was up to $25 \%$ smaller than the one for the equivalent disk. In the early 90s, Kajikawa (1992) studied the falling descent of 'snow crystals', showing that dendritic crystals with large internal ventilation fall following a stable descent over a larger $R e$ range compared to simple hexagonal plates. This result was later confirmed by Vincent et al. (2016), showing 
that the internal ventilation of disks improves the stability of the falling motion.

Despite these isolated studies, a gap still remains on how the wake of planar irregular particles differ from the characteristic wake of a disks. In here, we describe and compare the motion of a free falling disk with a square and a hexagonal planar particle with same frontal area and material properties. The surrounding flow is also investigated by means of volumetric velocimetry (3D-3C) so that the coupling between vortex shedding and particle motion can be addressed.

\section{Methods and experimental setup}

To investigate how the frontal geometry of a planar particle affects the settling dynamics and wake characteristics we manufactured planar particles with three different geometries. The reference particle is a disk with diameter $D=30 \mathrm{~mm}$, thickness $h=2 \mathrm{~mm}$ and density $\rho_{\mathrm{p}}=1.2 \mathrm{~g} /$ $\mathrm{cm}^{3}$ that lies within the fluttering regime, with a measured Reynolds number $R e \approx 1370$ and dimensionless moment of inertia $I^{*}=5 \times 10^{-3}$. Then, keeping the particle thickness $(h)$ and frontal area $\left(A_{\mathrm{p}}\right)$ constant we vary the geometry of the perimeter to a square and a hexagonal shape. Particles were laser cut within a precision of $\pm 0.5 \mathrm{~mm}$ and painted black to facilitate the image processing.

In water, the planar particles were released from about two disk diameters below the water surface so that entry and surface effects were avoided. The particles were always released using an active suction mechanism with a suction cup of $10 \mathrm{~mm}$ diameter that can accommodate all three geometries. The angle of the particle prior to the release was carefully adjusted to zero. To control the particle angle, the suction cup was mounted flushed into a submerged flat plate. Therefore, when the particle was being held by the suction cup the upper surface of the particle was parallel and in contact to the plate. The alignment of the submerged plate was carefully performed before filling the tank using a digital angle within an accuracy of $0.1^{\circ}$. This initial tilt angle accuracy is sufficient to have descent trajectories unaffected by this parameter, as shown in Lee et al. (2013) for thin disks within the same $R e-I^{*}$ region.

The water tank where the particles were released is an open glass tank of $0.45 \mathrm{~m}$ square cross section and 0.5 $\mathrm{m}$ high. To record the particle descent, two JAI-GO5000 cameras were used. One camera captured the frontal view of the descent motion, whereas the other captured the pla$\operatorname{nar}(X-Y)$ motion of the particle through a mirror at $45^{\circ}$ located underneath the water tank. The cameras were both focussed on the middle plane of the tank to minimise the lens curvature distortion. The trajectories were recorded at $60 \mathrm{fps}$ and this was sufficient to resolve the translational motion during all parts of the descent to within $2 \%$ of the particle diameter. In each frame the dark particle projection is recorded onto the white background and the position of the particle centre of mass was obtained by locating the geometric centre of each particle projection. The image processing was performed using an in-house script developed in MATLAB, as in Esteban et al. (2018) and Esteban et al. (2019). The method to obtain the particle position from the grey-scale image is a threshold-based method, where raw images are converted into black and white images after applying a user-defined intensity threshold. For the accuracy on the particle location we rely on a stable light intensity during all the trajectories. Commercial LED panels connected to a stable DC power supply were used to back illuminate the complete field of view of both cameras. The measured trajectories were smooth, and a polynomial filter of $3 \mathrm{rd}$ order and frame length of 5 points was used to filter out high-frequency noise.

A set of releases for a sphere falling in air was performed to establish limitations on the accuracy associated with the suction mechanism as well as the camera alignment. The variance in the landing position was interpreted as the uncertainty of the complete system. This was found to be two orders of magnitude smaller than the sphere diameter, in accordance with the uncertainty typically found in the literature for similar drop mechanisms, (Heisinger et al. 2014) among others. A square grid was placed inside the water tank to measure the camera magnification; and to account for image distortion from the lens in the interrogation area but this was found to be negligible.

To build a baseline for comparison with the square and hexagonal particles, a first set of drops for the circular disk was performed. The first section of the particle descent is known to be influenced by the initial transient dynamics, and the determination of the distance at which the particle reaches a 'steady state' is non-trivial (this term refers to the natural falling style of the particle once this is not influenced by initial conditions). Heisinger et al. (2014) showed experimentally that for disks with $I^{*}=3 \times 10^{-3}$ and $R e \approx 1200$ a vertical distance of about $6.5 D$ from the release point was required to achieve a steady state. Here the reference disk has similar dimensionless parameters as in Esteban et al. (2018) $\left(R e \approx 1370, I^{*} \approx 5 \times 10^{-3}\right)$, and therefore we use the same distance of $7 D$ as the start of the steady state of all particles tested in this study.

The bottom of the tank also influences the dynamics of the particles due to hydrodynamic interactions and therefore we do not process the particle trajectory once it reaches a distance of $2 D$ from the tank bottom. Thus, we use trajectory sections that go from $7 D$ from the top to $2 D$ from the bottom, and these are not influenced neither by initial nor end conditions. 
Particles are always released in water at room temperature $\rho_{\mathrm{f}}=0.998 \mathrm{~g} / \mathrm{cm}^{3}$ and $v=1.004 \times 10^{-6} \mathrm{~m}^{2} / \mathrm{s}$. The waiting time between successive drops was $20 \mathrm{~min}$, corresponding to more than 600 times the particle time-scale of the oscillatory motion. This waiting time was verified to be sufficient to have quiescent flow by visualizing tracer particles with the a volumetric 3-component velocimetry system (V3V camera system from TSI).

The water media was seeded with $55 \mu \mathrm{m}$ polycrystalline particles. Then, using the same approach, another set of 15 trajectories was recorded at $7.25 \mathrm{fps}$ and these were synchronised with the V3V camera system. The volumetric measurements of the instantaneous velocity field were obtained for a vertical location where the particle trajectory was steady ( $>10 D$ from the release point). This camera system consists of three $2048 \times 2048$ pixels 12 -bit frame-straddle CCD cameras aligned in a coplanar triangle pattern. This configuration allowed us to map a field of view (FOV) of $140 \mathrm{~mm} \times 140 \mathrm{~mm} \times 60 \mathrm{~mm}$, as sketched in Fig. 1. A synchroniser by TSI was used as an external trigger and connected to the laser and the camera system. A $200 \mathrm{~mJ} /$ pulse double-pulsed laser (Bernoulli-PIV Litron) was used to illuminate the FOV. The two-frame double exposure image pairs were set at $7.25 \mathrm{~Hz}$ and image triplets were analysed via Insight $4 \mathrm{G}$ software from TSI. The $3 \mathrm{D}$ imaging principle of this system is a multi-view photo-grammetry technique. The FOV of the three cameras intersect to form the camera system mapping region. Thus, any seeding particle inside the FOV is recorded from three different angles creating the basis for multi-view stereo vision. In the image plane, the three particle images form the coplanar camera arrangement form a triangle, which centre determines the $x$ and $y$ coordinates of the particle. The defocusing of the triangle formed is the basis to obtain the location in the $z$ coordinate

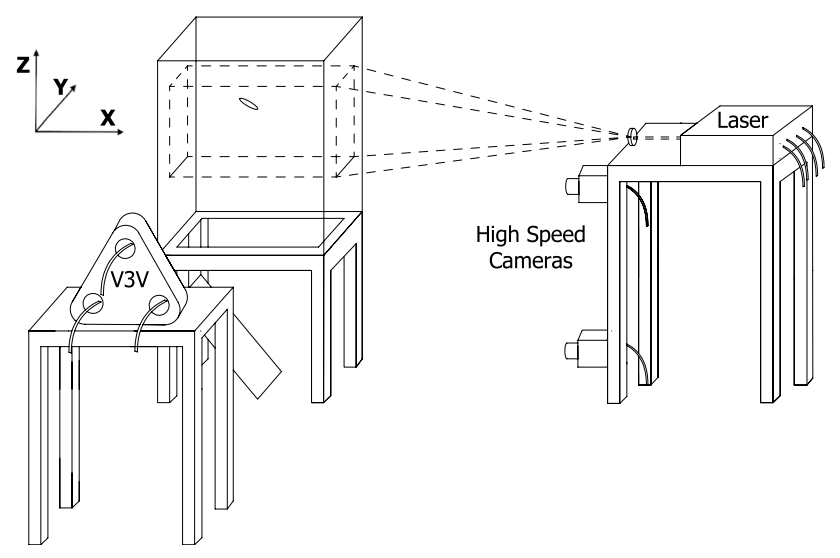

Fig. 1 Sketch of the experimental setup. Two high-speed cameras record the trajectory of the particle (front view and bottom view through a mirror at $45^{\circ}$ ). The $\mathrm{V} 3 \mathrm{~V}$ system acquires triplets of images that are later used to reconstruct the velocity field around the falling particle from the reference plane where the three views overlap. The reader is referred to Pothos et al. (2009) for a more detailed explanation of the imaging and processing principles of this technique.

In these experiments we performed a pre-calibration before each set of drops. This was done using a backlighted calibration target with dimensions of $140 \times 140 \mathrm{~mm}^{2}$ with a Cartesian grid of circular dots with a distance of $2 \mathrm{~mm}$ in both directions. A traverse system was used to move the calibration target to different positions along the camera system optical axis. The maximum distance between these planes was $2 \mathrm{~mm}$ and a total number of 30 planes were recorded.

The de-warping error is also obtained from the calibration. This is a measure of the standard deviation of grid dot position error after de-warping and was found to be $\leq 0.3$ pixels. Considering that tracer displacements were limited to less than 8 pixels, the spatial uncertainty in the flow velocimetry is about $4 \%$. Also, the magnification of the cameras was compared to the pinhole camera at each calibration plane and this is used to adjust the pixel size in each of the three views during the triplet search.

A node volume of $10 \mathrm{~mm}^{3}$ was defined with a $75 \%$ of node volume overlap in the velocity interpolation process. The smoothing factor is set such as velocity information from neighbouring nodes do not contribute to the current node volume. The FOV of the V3V system was set after a vertical distance of $7 D$ from the release point to assure the steady state of the particle. For each particle, 15 trajectories were recorded and then fully processed and reviewed to assure the robustness of the results. During these observations, particles were found to describe both zig-zag and three dimensional descents but we always found that similar particle trajectories shared the same wake characteristics.

\section{Determination of non-dimensional parameters}

Five dimensional quantities are generally used to define the non-dimensional parameters that characterise the falling style of disks and quasi $2 D$ rectangular plates; and these quantities are: the characteristic length, the thickness and the density of the particle; and the density and kinematic viscosity of the fluid. These dimensional quantities are combined to form three dimensionless numbers that determine the settling behaviour of the freely falling particle. These are the dimensionless moment of inertia $\left(I^{*}\right)$, the Reynolds number $(R e)$ and the aspect ratio of the particle $(\chi)$. The planar particles of this study have a nearly constant aspect ratio, $\chi \approx 0.07$. Therefore, we believe the dynamics of the fall to be independent of this parameter. However, the particles have severe differences in their frontal geometry and the particle characteristic length cannot be fully captured by 
the equivalent particle diameter. Thus, we use the approach proposed in Esteban et al. (2018) to obtain a new particle characteristic length-scale from which to obtain an equivalent Reynolds number and dimensionless moment of inertia. Thus, the dimensionless moment of inertia is defined as

$I^{*}=\frac{I_{\mathrm{p}}}{\rho_{f} \ell^{5}}$

where $\ell=D_{\mathrm{c}} / Q$, with $D_{\mathrm{c}}$ the diameter of the circumscribed circle and $Q=4 \pi A_{p} / P^{2}$ is the isoperimetric quotient. Similarly, the Reynolds number is defined as,

$R e=\frac{\ell\left\langle V_{\mathrm{z}}\right\rangle}{v}$.

The advantage of using this definition for the particle characteristic length-scale, $\ell=D_{\mathrm{c}} / Q$ is that the characteristic length $(\ell)$ of the reference disk is still its true diameter and therefore the $\left(R e-I^{*}\right)$ phase diagram defined by Field et al. (1977) is not altered. The Reynolds number and dimensionless moment of inertia for disks, hexagons and squares studied in here appear to be within the fluttering regime originally defined by Field et al. (1977) (Fig. 2). The dimensionless numbers of these particles can be found in Table 2 .

\section{Results}

In this section, results on the trajectory characteristics and flow visualization of the wake behind the planar particles are presented. First, we introduce the trajectory characteristics obtained from the high-speed cameras and the differences between frontal geometries are compared. Second, vorticity iso-surfaces and contours in the wake of the particles are shown for trajectories that are representative of the motion of each geometry.

\subsection{Trajectory characteristics}

Two views of the particle descent are acquired synchronously so that the $3 \mathrm{D}$ position of the particle can be obtained, as in Fig. 3. We observe that different trajectories show strong differences in the relevance of the out-of-plane motion, as observed experimentally and in simulations for disks with different dimensionless inertia $I^{*}$ [Zhong et al. 2011, 2013; Zhong and Lee 2012; Chrust et al. 2013; Auguste et al. 2013 among others] and experimentally for planar polygon particles (Esteban et al. 2018). Thus, in here 'planar zig-zag' motion refers to trajectories with two trajectory sections that repeat periodically; gliding sections where the particle oscillates within a $X-Y$ plane and turning sections where the particle shows small
Table 1 Geometric characteristics of the particles; $A_{\mathrm{p}}$ refers to frontal area, $D_{\mathrm{c}}$ to diameter of circumscribed disk, $P$ to perimeter of frontal geometry and $Q$ to isoperimetric quotient, defined as $Q=4 \pi A_{\mathrm{p}} / P^{2}$. Mean descent velocity $\left(\left\langle V_{\mathrm{z}}\right\rangle\right)$ and peak to peak velocity $\left(V_{\mathrm{z} p p}\right)$ along the descent. The (zls) notation in the hexagons stands for particles describing zig-zag and spiral, respectively

\begin{tabular}{llll}
\hline & Disk & Hexagons (zls) & Square \\
\hline$A_{\mathrm{p}}\left(\mathrm{cm}^{2}\right)$ & 4.34 & 4.39 & 4.41 \\
$D_{\mathrm{c}}(\mathrm{cm})$ & 2.35 & 2.6 & 2.97 \\
$P(\mathrm{~cm})$ & 7.4 & 7.8 & 8.4 \\
$Q$ & 1 & 0.91 & 0.79 \\
$\operatorname{Mass}(\mathrm{g})$ & 1.07 & 1.01 & 1 \\
$\left\langle V_{\mathrm{z}}\right\rangle\left(\mathrm{mm} \cdot \mathrm{s}^{-1}\right)$ & $58 \pm 2$ & $56 \pm 3 \mid 54 \pm 2$ & $55 \pm 3$ \\
$V_{\mathrm{z} p}\left(\mathrm{~mm} \cdot \mathrm{s}^{-1}\right)$ & 110 & $94 \mid 78$ & 67 \\
\hline
\end{tabular}

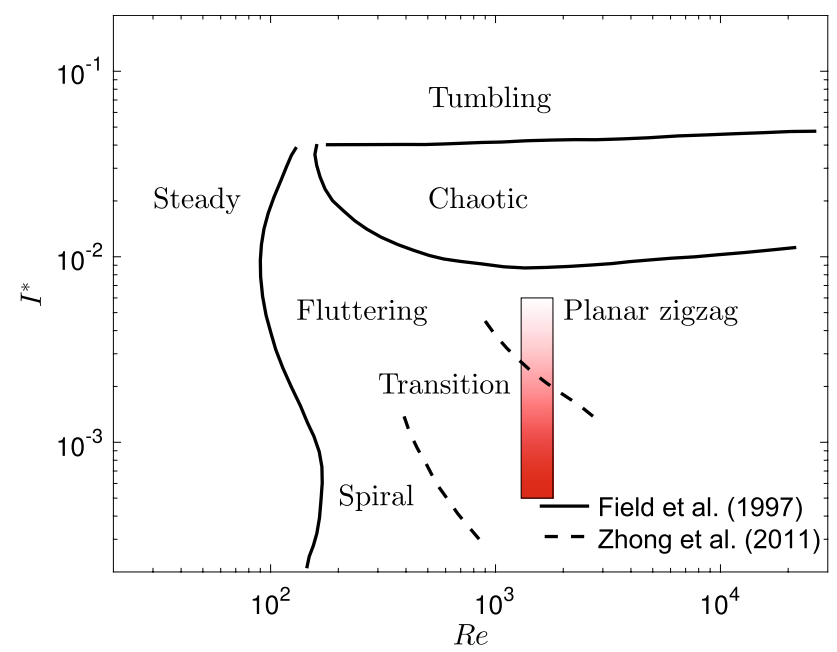

Fig. $2 R e-I^{*}$ phase diagram for thin circular disks as in Esteban et al. (2018). The solid and broken lines define the regimes and subregimes found in Field et al. (1977) and Zhong et al. (2011), respectively. The colour gradient is a qualitative measure of the likelihood of any particle to describe either 'planar zig-zag' or 'spiral' motion, where white represents pure 'planar zig-zag' motion and dark red 'spiral' motion. A more detailed analysis on the statistics of the particle trajectory can be found in Esteban et al. (2018)

deviations from the idealised complete turn of $180 \mathrm{deg}$ in planar velocity direction. Similarly, 'transitional' motion refers to trajectories with the same oscillatory pattern as in 'planar zig-zag' motion but with a superimposed constant angular velocity about the $Z$-axis. The particle trajectory in the $X-Y$ plane resembles a rhodonea curve, as shown in Zhong et al. (2011) for disks with small dimensionless moment of inertia $I^{*}$. Trajectories describing highly 3D motion are named 'spiral' motion; the $X-Y$ footprint of these trajectories shows a particle describing a sequence of circles with a finite offset between consecutive loops. These trajectories also show a much steadier descent 


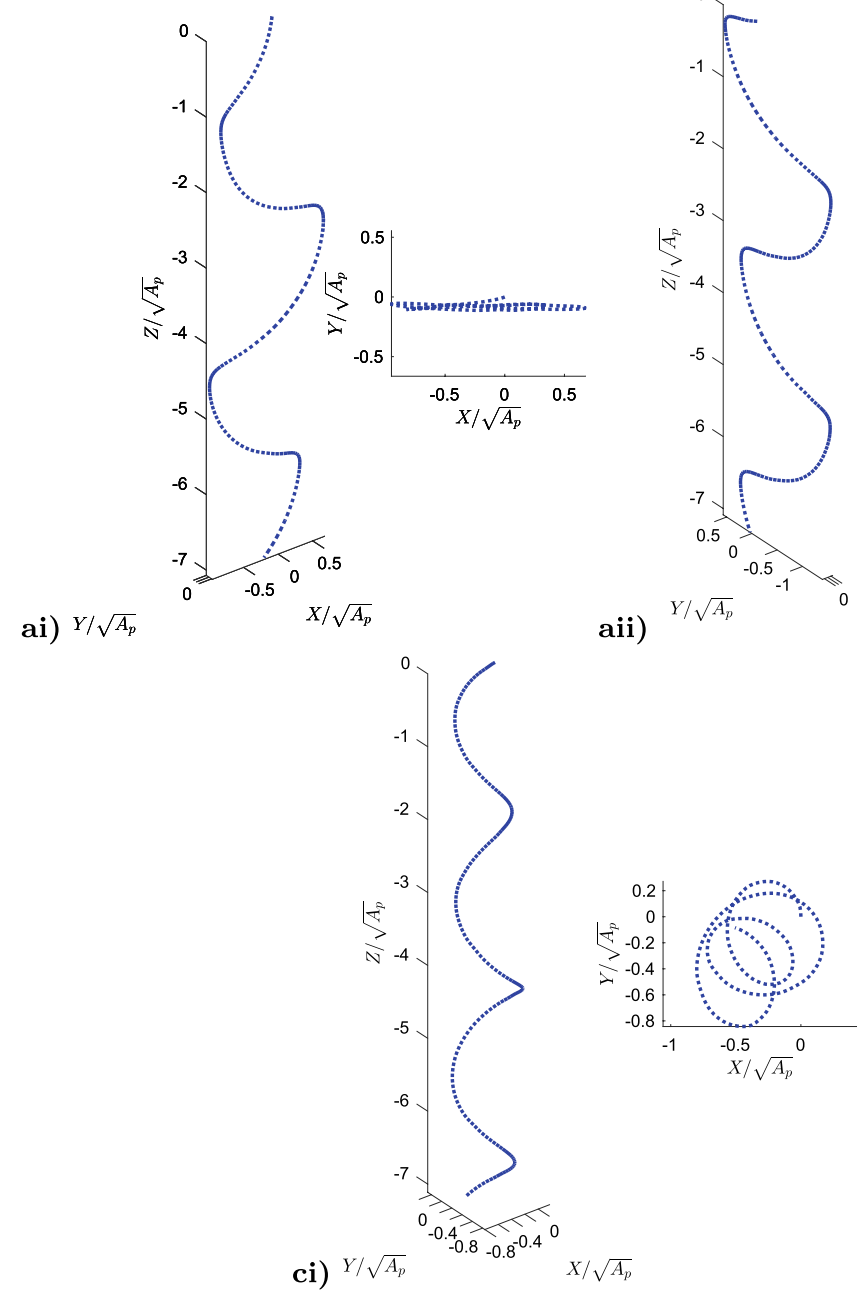

Fig. 3 Reconstructed trajectory section of planar particles describing the three regimes proposed in Esteban et al. (2018); a, (i) disk under 'planar zig-zag' motion, a, (ii) hexagon under 'planar zig-zag'

velocity. This is captured in the peak to peak velocity $\left(V_{\mathrm{z} \mathrm{p} p}\right)$ shown in Table 1.

The variation in the mean descent velocity measured from the experimental data for the three geometries can be explained in terms of the variations in mass and surface area $A_{p}$. From a force balance between gravity forces and aerodynamic forces, the average descent velocity $\left\langle V_{\mathrm{z}}\right\rangle$ is expected to scale with $\sqrt{M g / \rho A_{\mathrm{p}}}$. If one computes the nondimensional descent velocity for each geometry using the measured physical properties of the particles they are within $1 \%$ of each other. This suggests that variations in $Q$ are unlikely to cause variations in the mean descent velocity of the particles. This is consistent with our previous observations where $Q$ plays an important role in determining the non-dimensional inertia of the object and not necessarily the descent velocity (or the corresponding Reynolds number).
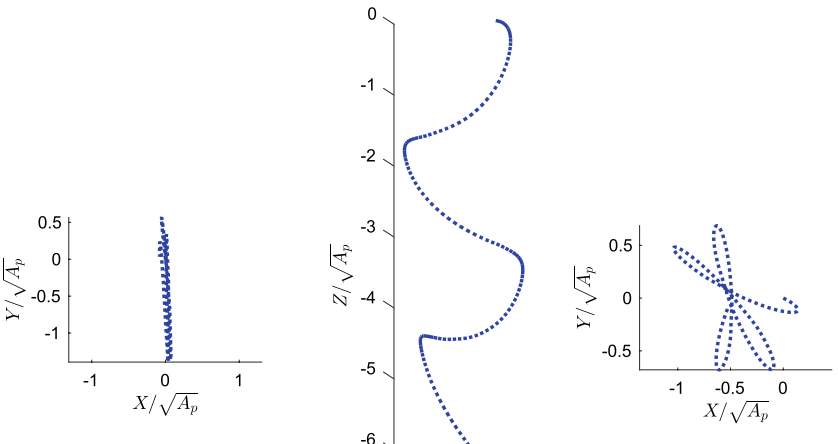

$X / \sqrt{A_{p}}$

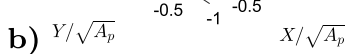

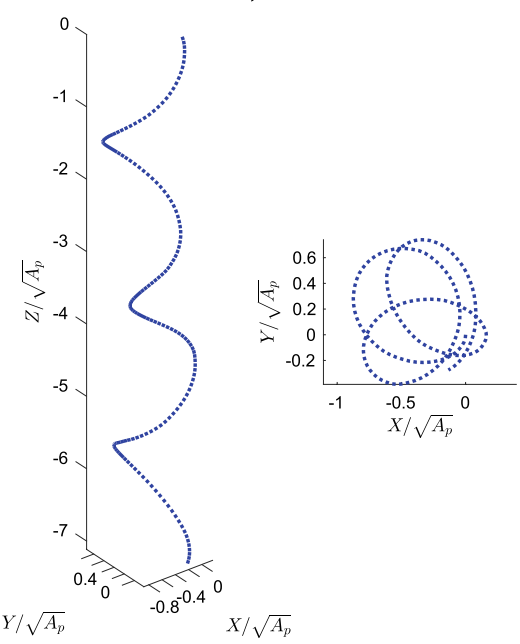

motion, b hexagon under 'transitional' motion, c, (i) hexagon under 'spiral' motion and c, (ii) square under 'spiral' motion

We observe a difference in descent velocity between hexagons describing 'zig-zag' and 'spiral'. The descent velocity is about 5\% higher for the particles describing 'zig-zag' motion but this result comes from a small number of drops. We believe that a more thorough experiment (multiple planar particles with different Reynolds numbers) should be done to establish a more robust relationship between falling

Table 2 Values of the particle Reynolds number $(R e)$ based on the mean fall velocity $\left(\left\langle V_{\mathrm{z}}\right\rangle\right)$ and the characteristic length-scale $(\ell)$, and dimensionless moment of inertia $I_{\ell}^{*}$ based on the length-scale $(\ell)$

\begin{tabular}{llll}
\hline & Disk & Hexagon & Square \\
\hline$I_{\ell}^{*}$ & $5.01 \cdot 10^{-3}$ & $1.95 \cdot 10^{-3}$ & $5.13 \cdot 10^{-4}$ \\
$R e_{\ell}$ & 1370 & 1450 & 1620 \\
\hline
\end{tabular}


style and mean descent velocity, as performed in Tam (2015) for elastic plates.

Square plates show a peak-to-peak velocity along the trajectory $\left(V_{\mathrm{z} p p}\right)$ approximately $30 \%$ smaller than disks, showing that the descent motion of these particles is much steadier than that observed in disks.

In Lee et al. (2013), they described how a non-uniform lift distribution over the disk surface can create a liftinduced torque, leading to the disruption of the original planar motion. Following the same reasoning they showed how the dimensionless moment of inertia of the particle $\left(I^{*}\right)$ might either stabilise or destabilise the system depending on its relative magnitude. Therefore, if $I^{*}<I_{\text {crit }}^{*}$ the system instabilities grow at every turning point, while if $I^{*}>I_{\text {crit }}^{*}$ the system becomes more stable. The non-uniform lift distribution explained in Lee et al. (2013) occurs naturally for particles with non-axisymmetric frontal geometries. We hypothesize that this mechanism is the reason why as the particle circularity reduces the likelihood of having a planar trajectory also does, as shown in Esteban et al. (2018) and confirmed here.

In the following section, the strong differences in the falling dynamics between particle trajectories are investigated by means of volumetric flow analysis of the wakes.

\subsection{Wake characteristics}

The effect of vorticity in the flow behind the particle is one of the key features to understand the path instability of planar particles. Disks freely falling in viscous media have been extensively investigated during the last 60 years, as in Willmarth et al. (1964), Field et al. (1977), Fernandes et al. (2005), Zhong et al. (2011) or Lee et al. (2013) among others. Fernandes et al. (2005) showed experimentally that the phase difference between the velocity and the inclination of the body axis greatly differs for the irrotational theory estimation, showing that vortical effects in the wake were crucial to understand body dynamics. A few years later, Zhong et al. (2011) showed that thin disks lying in the $R e-I^{*}$ domain corresponding to fluttering motion exhibit three different descent styles that are associated with a change in their dimensionless moment of inertia $\left(I^{*}\right)$; i.e. 'planar zigzag', 'transitional', and 'spiral'. They used fluorescence dye to visualized vortex patterns corresponding to the 'planar zig-zag' and 'spiral' descent. This showed that in each cycle of the zig-zag motion a pair of hairpin vortices were shed into the wake, whereas for the spiral descent they observed a helicoidal vortex evolution. Similarly, Lee et al. (2013) performed flow visualizations with fluorescence dye and planar PIV measurements for disks describing a 'zigzag-spiralzigzag' intermittence finding that the characteristic turbulent structures in the wake of the particle go hand by hand with their descent mode. Thus, disks describing zig-zag descent create a vortex loop elongating in the direction of the body movement to form hairpin-like structures, but as the planar symmetry is broken, disks shed a vortex chain from the outer edge, forming a helicoidal vortex wrapping around the wake region.

To the best knowledge of the authors, this is the first experimental study showing three dimensional three component flow fields in the wake of planar particles. Also, it shows the strong connection between particle descent style and wake characteristics for the fluttering sub-modes identified in Zhong et al. (2011) ('planar zig-zag', 'transitional' and 'spiral' motion) but extended to other planar geometries.

\subsubsection{Disks}

We found severe differences in the wake of a particle describing 'planar zig-zag' motion depending on its location relative to the turning point. The wake behind a disk describing 'planar zig-zag' motion shows characteristic vortex structures that repeat during the particle descent at every gliding and turning section. As the disk describes a gliding section of the trajectory, a pair of counter-rotating vortices progressively forms in the downstream direction from both sides of the symmetry plane of the disk aligned with the gliding motion. These vortices remain primarily aligned with the motion of the disk, as shown in Fig. 4. On the other hand, as the disk approaches a turning event, it decelerates and increases angle of attack. When the angle of attack becomes too high the disk experiences stall and a recirculation zone, whose predominant vorticity component is normal to the direction of motion, is formed at the leading edge of the disk (shown in red in the iso-contours). Then, the particle planar velocity reduces progressively together with the descent velocity up to the turning point where it becomes zero and the disk motion reverses direction. At this point, the recirculation zone at the leading edge has grown in size and detaches forming a hairpin-like vortex. The development of the vorticity structures on the circular disk leading edge near the turning point resembles the leading edge vortex observed in flapping foils, where this structure is also shed into the wake after the motion is reversed. These measurements confirm the wake structures visualized by Zhong et al. (2011) with fluorescence dye. On the other hand, these measurements do not show the second recirculation zone found in Lee et al. (2013) on the lower surface of the particle. Lee et al. (2013) showed that the both recirculation zones coexisted at a similar relative location of the trajectory and eventually merged together and detached also forming a hairpin-like vortex. We believe that the lower recirculation zone might form and develop during short times and the limited time resolution of the system used in here (7.25 fps) might not be sufficient to capture this wake dynamics. Similarly, we do not capture secondary hairpins in the wake 
Fig. 4 a Reconstructed trajectory section of a disk describing 'planar zig-zag' motion with vorticity iso-surfaces of the wake behind the disk at different locations relative to the turning point. b Contour plots of the vorticity magnitude in the $X-Y$ plane at a distance of $\sqrt{A_{p}}$ from the upper surface of the particle at the same locations. The iso-surfaces have a magnitude of $2.5 \times 10^{-3} \mathrm{~s}^{-1}$ and the contours are also saturated at $2.5 \times 10^{-3} \mathrm{~s}^{-1}$. Yellow and blue contours represent positive and negative $X$-vorticity, black and red $Y$-vorticity, green and pink $Z$-vorticity
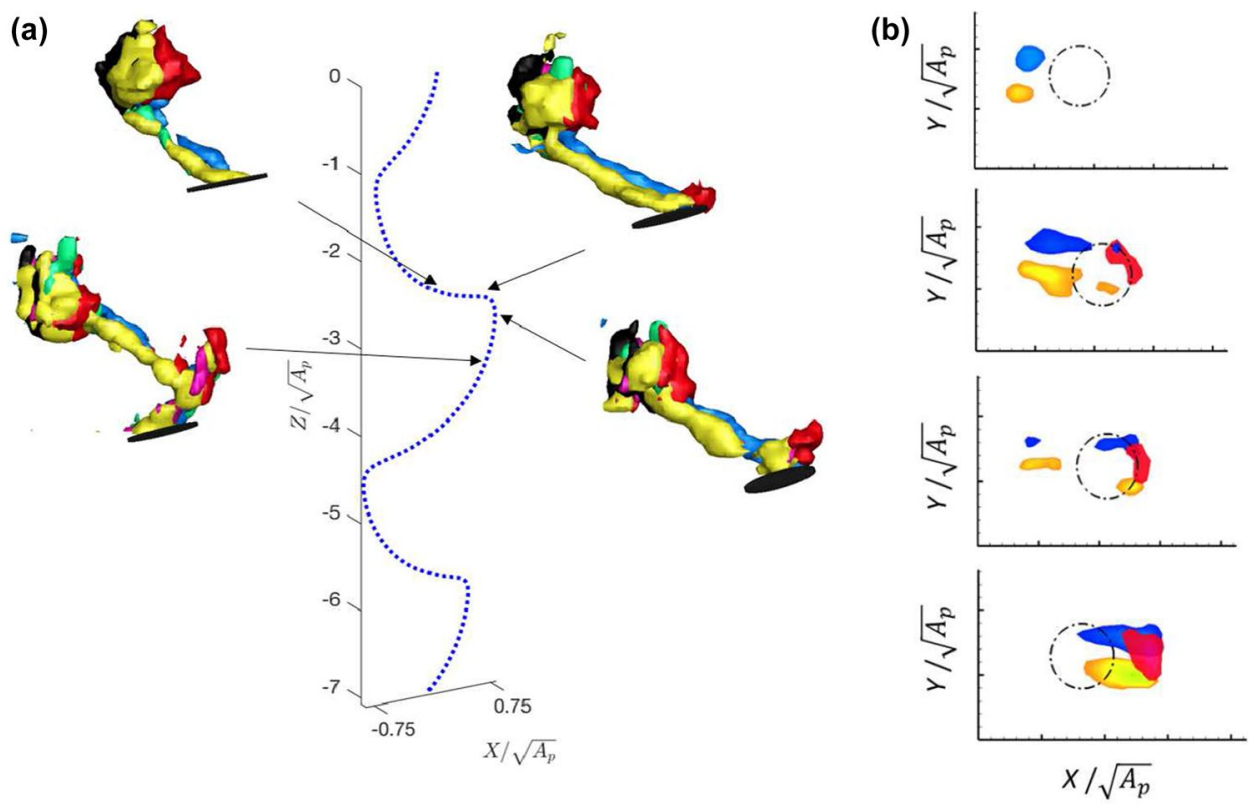

of the particle. We believe this is due to the low vorticity magnitude that these structures carry relative to the counterrotating vortices that grow from the leading edge of the disk, as described in Zhong et al. (2013).

The complete wake region of the disk now includes two rows of hairpin-like vortices evenly spaced in the vertical direction that carry vorticity in opposite directions linked by a pair of counter rotating vortices. The overall structure of the wake is also in good agreement with results from numerical simulations of free-falling thin disks at moderate Reynolds numbers, as in Churst et al. (2013). A closer look to the vortical structures shed off at every extreme point of the oscillatory motion shows that these are responsible for the regions of strong downwash in the wake of the disk. The magnitude of the velocity in the region dominated by the downwash is of the same order of magnitude of the descent velocity observed along the disk trajectory and this also agrees with previous planar particle image velocimetry data in Zhong et al. (2013).

\subsubsection{Square plates}

The wake characteristics behind a square plate falling under 'spiral' motion are almost unaffected by the relative position of the particle along the trajectory, exhibiting nearly continuous shedding of vortical structures along the perimeter of the particle. The vortex structures are significantly different from those observed in the planar motion of a disk. Here, vortex structures with different dominant vorticity components are formed indistinctly during the descent of the particle, as in Fig. 5a. Each of the particle corners perceive the flow with a different relative angle of incidence and therefore generate vortical structures with different dominant components. Taken together, these different vorticity components give rise to a large-scale vortex structure in the wake during the particle fall. This is best visualized by examining the contours in the wake (shown in separate contour plots on the right) taken at one characteristic length scale $\left(\sqrt{A_{p}}\right)$ behind the particle. These contours clearly show that there is really no difference in the strength of vorticity or a preference to which side of the particle this vorticity is present. It almost seems like the vorticity has rotational invariance about the vertical axis, despite the fact that the particle itself is not axisymmetric. This invariance in the vorticity strength is a reflection of the type of motion followed by the square plates. When the square describes a section of the trajectory with any of its corners aligned with the direction of motion, a pair of counter-rotating vortices forms from both sides of the corner, propagating downstream (as in the leading edge of a delta wing). However, instabilities in the particle wake due to small misalignments of the leading corner with the lateral motion or instabilities originated at other particle sharp corners quickly realign the particle to a different orientation. In this new orientation, the same phenomenon is repeated. Therefore, the descent of a square particle becomes a series of reorientations all along its trajectory. This process results in the breaking down of individual vortical structures and gives rise to a wake with nearly rotational invariance. Further time-resolved measurements with higher spatial resolution are necessary to examine the finer details of this wake formation. 
Fig. 5 a Reconstructed trajectory section of a square describing 'spiral' motion with vorticity iso-surfaces of the wake behind the square at different locations. b Contour plots of the vorticity magnitude in the $X-Y$ plane at a distance of $\sqrt{A_{\mathrm{p}}}$ from the upper surface of the particle at the same locations. The iso-surfaces have a magnitude of $2.5 \times 10^{-3} \mathrm{~s}^{-1}$ and the contours are also saturated at $2.5 \times 10^{-3} \mathrm{~s}^{-1}$. Yellow and blue contours represent positive and negative $X$-vorticity, black and red $Y$-vorticity, green and pink $Z$-vorticity
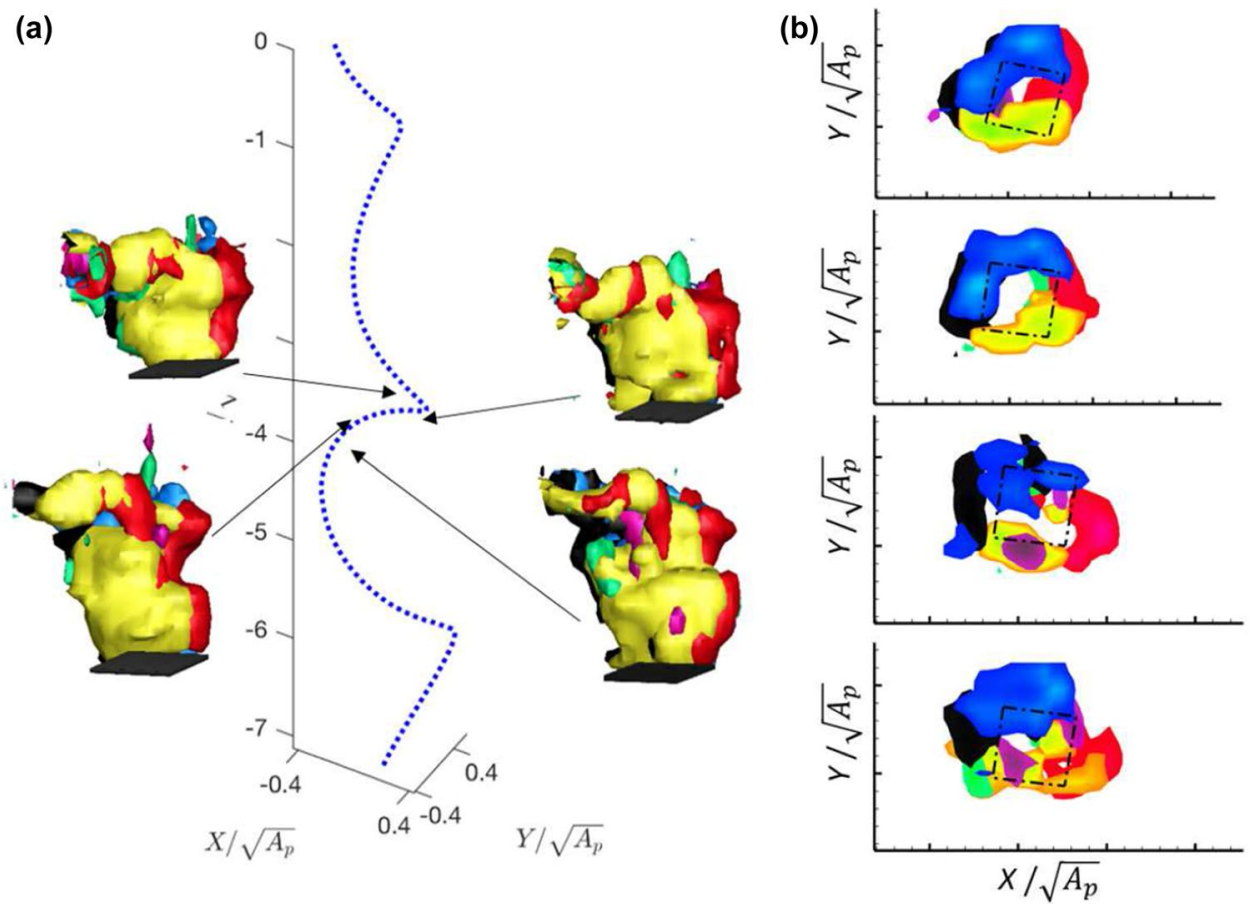

\subsubsection{Hexagons}

Freely falling hexagons describe both types of trajectories; i.e. 'planar zig-zag' and 'spiral' motion. When the hexagon describes 'planar zig-zag' motion, the wake behind the particle has some similarities to the wake behind disks under 'planar zig-zag', as seen in Fig. 6a. The vortex structures shown for disks seem to be present in the wake of the hexagon but the strength of these structures for the hexagons is weaker and therefore not as clearly observed in the isosurfaces. However, the contour plots taken downstream of the particle in Fig. 6b seem to show that the vorticity is preferentially distributed along the perimeter of the particle just as in the case of the disks. As the hexagon describes a gliding section of the trajectory, two large counter-rotating vortices appear on both sides of the symmetry plane of the hexagon aligned with the motion, yet they are substantially altered by the vorticity generated on the other edges of the
Fig. 6 a Reconstructed trajectory section of a hexagon describing 'planar zig-zag' motion with vorticity isosurfaces of the wake behind the hexagon at different locations relative to the turning point. $\mathbf{b}$ Contour plots of the vorticity magnitude in the $X-Y$ plane at a distance of $\sqrt{A_{p}}$ from the upper surface of the particle at the same locations. The iso-surfaces have a magnitude of $2.5 \times 10^{-3} \mathrm{~s}^{-1}$ and the contours are also saturated at $2.5 \times 10^{-3} \mathrm{~s}^{-1}$. Yellow and blue contours represent positive and negative $X$-vorticity, black and red $Y$-vorticity, green and pink $Z$-vorticity
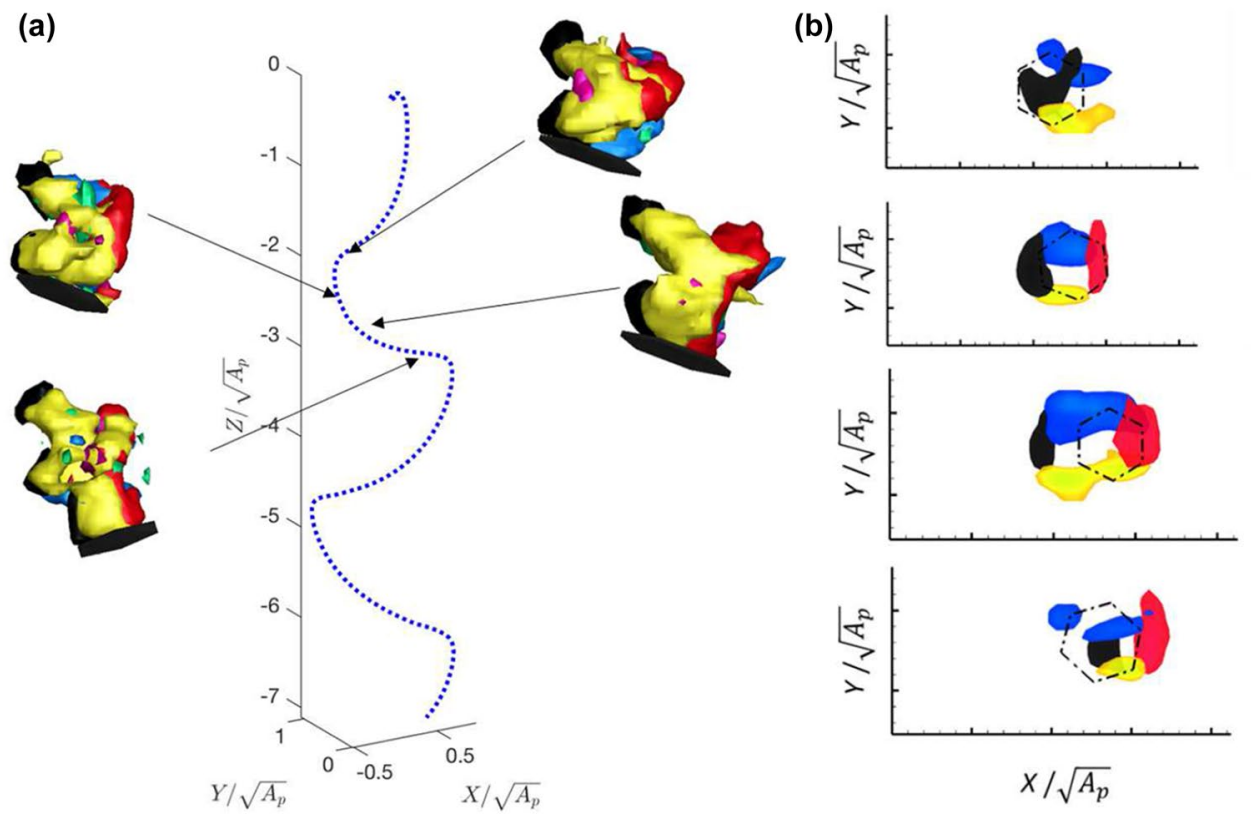
geometry. It is hypothesised that when the particle reaches a turning point, the non-uniform pressure distribution along the particle geometry is the reason why the particle rotates about an axis perpendicular to the particle surface and it is realigned towards a different plane of motion. Thus, the hexagon starts a new cycle, following a 'planar zig-zag' motion but contained in a new oscillatory plane. However, when the hexagon describes 'spiral' descent, see Fig. 7a), the wake of the particle resembles the wake shown for square plates, with similar vorticity distribution along the particle descent. This is also clear in the contour plots, shown in Fig. 7b), obtained downstream of the particle and the similarities with the wake of a square are clear.

Thus, particles with different frontal geometry; i.e. disks and hexagons; and hexagons and squares, exhibit qualitatively similar wake structures when falling under the same mode. However, the modes of secondary motion for the same conditions are not equally probable for all particles, but depend on the particle geometry.

To have a more quantitative comparison between the wakes of the particles, Fig. 8 shows the probability density function (PDF) of the non-dimensional vorticity magnitude $\left(\tilde{\omega}=\omega V_{\mathrm{z}} / \sqrt{A_{p}}\right.$, where $\left.\omega=\sqrt{\omega_{x}^{2}+\omega_{y}^{2}+\omega_{z}^{2}}\right)$ on the wake of the $\mathrm{n}$-sided polygons investigated. Since the vorticity in the wake of particles has different components and direction, examining the distribution of vorticity magnitude provides a way to compare these across all cases. The three dimensional vorticity magnitude fields used for this task contain a fluid domain that includes two particle oscillations about the mean descent velocity. The velocity fields are filtered prior to computing vorticity magnitude using a Gaussian filter with kernel size of half of the diameter of the reference circular disk.

The distribution of the vorticity magnitude in Fig. 8a shows a progressive decrease in high-vorticity events in the wake of the particle as this becomes less axisymmetric, where black corresponds to disks, green to hexagonal particles and red to squares. To evaluate the connection between falling style and the signature of vorticity magnitude, we separate the trajectories of the hexagonal particles according to the descent style; i.e. 'planar zig-zag' and 'spiral' motion. Thus, we now obtain the distribution of vorticity magnitude for the hexagonal particles with similar trajectory style and wake morphology. Figure $8 \mathrm{~b}$ ) shows the non-dimensional vorticity magnitude of these two types of trajectories (green dashed line for hexagons in 'planar zig-zag' and green dotted line for hexagons in 'spiral') together with disks and squares. One can observe that the PDF for hexagonal particles describing 'planar zig-zag' motion becomes nearly identical to the one corresponding to disks, whereas the distribution in the vorticity magnitude in the wake of hexagonal particles describing 'spiral' motion resembles the one measured for square particles.

This suggests that the vorticity magnitude in the wake of planar particles with different frontal geometry might be well predicted once the falling style is known; and this at the same time can be approximated using the isoperimetric
Fig. 7 a Reconstructed trajectory section of a hexagon describing 'spiral' motion with vorticity iso-surfaces of the wake behind the hexagon at different locations. b Contour plots of the vorticity magnitude in the $X-Y$ plane at a distance of $\sqrt{A_{p}}$ from the upper surface of the particle at the same locations. The iso-surfaces have a magnitude of $2.5 \times 10^{-3} \mathrm{~s}^{-1}$ and the contours are also saturated at $2.5 \times 10^{-3} \mathrm{~s}^{-1}$. Yellow and blue contours represent positive and negative $X$-vorticity, black and red $Y$-vorticity, green and pink $Z$-vorticity
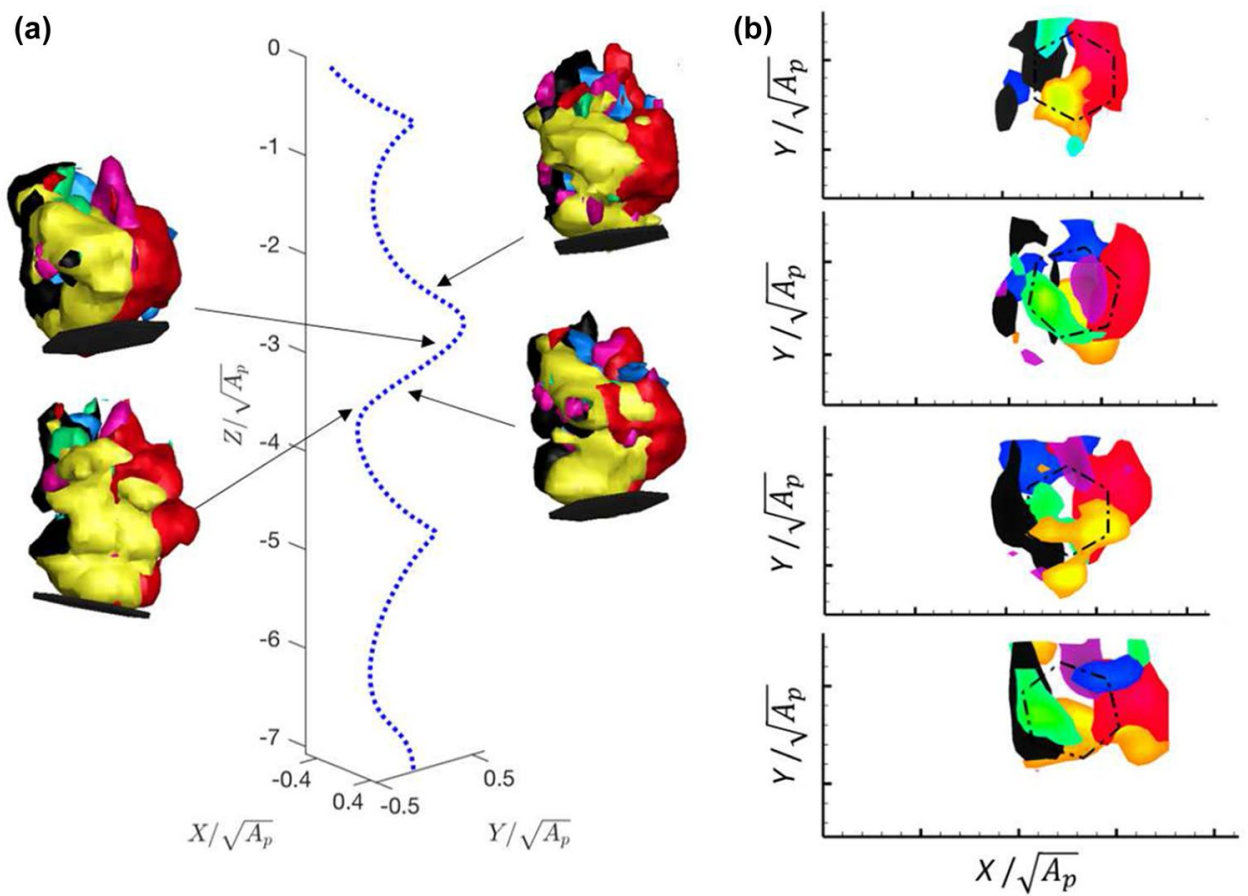


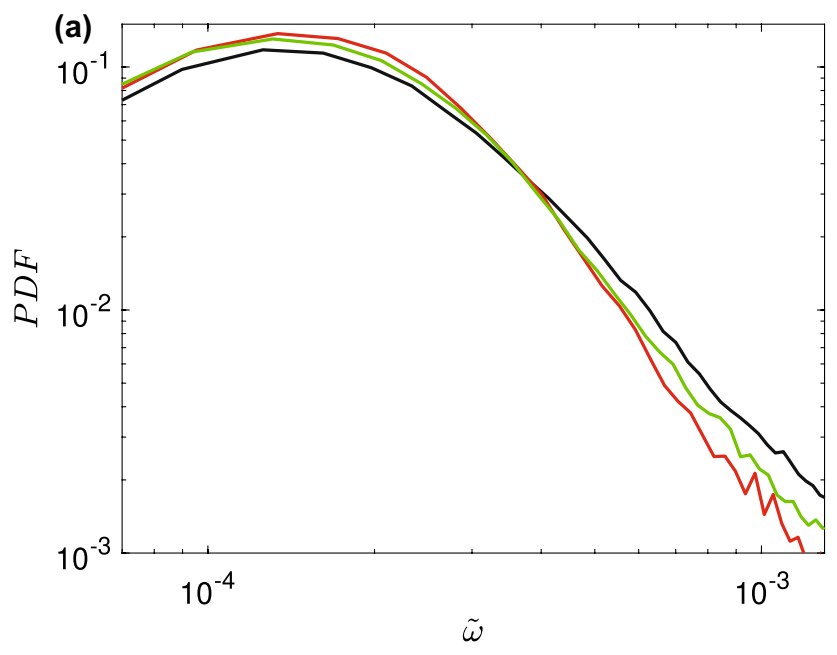

Fig. 8 a Probability density function of the non-dimensional vorticity magnitude in the wake of disks, squares and hexagonal plates. b Probability density function of the non-dimensional vorticity magnitude in the wake of disks, squares and hexagon plates grouped according to the descent style. The black solid line corresponds to

quotient of the frontal area, as discussed in Esteban et al. (2018).

\section{Conclusion}

This work investigated the effect of the particle edge geometry on the free-falling motion and associated wake characteristics of planar particles in a viscous media. The reference particle was chosen to be a disk that lies within the Reynolds - dimensionless moment of inertia domain $\left(R e-I^{*}\right)$ corresponding to the 'planar zig-zag' sub-mode found by Zhong et al. (2011) within the fluttering regime originally defined by Willmarth et al. (1964). The material properties and the frontal area of the planar particles were maintained constant and the isoperimetric quotient (a measure of the particle circularity) was varied by altering frontal geometry of the particle to different n-sided polygons; i.e. hexagons and squares.

High-speed imaging and three dimensional three component measurements of the instantaneous velocity field were used to characterise the particle trajectory and the associated wake. Disks were found to describe 'planar zig-zag' trajectories most of the time. These trajectories are characterised by a sequence of gliding-turning sections. The near wake of disks during the gliding phase is comprised by a pair of counter-rotating vortices that grow from the leading edge of the disk in the downstream direction carrying most of the vorticity in the stream-wise direction. In contrast, the near wake during the turning section is characterised by leading edge flow separation whose predominant vorticity is along

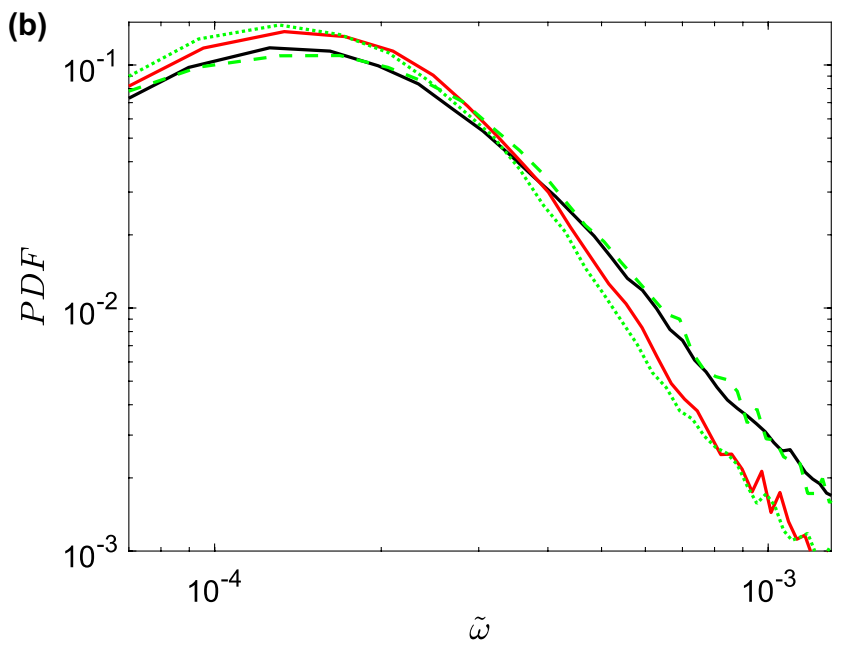

disks, the red solid line to squares, the green solid line to hexagonal planes prior to the trajectory classification, whereas the green dash line and the green dotted line correspond to hexagonal plates falling in 'planar zig-zag' and 'spiral' motion, respectively

the normal direction of motion. Thus, when the motion of the disk is reversed the recirculation zone on the upper surface detaches forming a hairpin-like vortex structure that is shed into the wake.

In contrast, squares were found to describe 'spiral' motion more often, with a wake dominated by large-scale shedding of vorticity along the entire periphery of the particle. The authors believe that the lack of a preference direction in the vorticity leads to the characteristic three dimensional trajectory of these particles. Relatively strong vortical structures are observed to the sides of a sharp corner when this is aligned with the particle planar motion. However, other vortical structures with different dominant components quickly realign the particle to a different orientation breaking the trajectory periodicity along the descent.

Hexagons exhibit 'planar zig-zag', 'transitional' and 'spiral' motions. When these undergo a 'planar zig-zag' motion their wake is qualitatively similar to the wake of a circular disk and for 'spiral' motions the wake exhibits large-scale vortex shedding as seen for square particles.

The probability density function of the non-dimensional vorticity magnitude in the wake of the particles shows that n-sided planar particles not only share similar descent style and wake morphology but also have wakes with comparable vorticity strength. However, these observations are limited to large-scale structures and noticeable differences in the wake of these particles might occur at smaller scales than half of particle diameter (kernel size of the Gaussian filter).

Further time-resolved measurements would be of great interest since these would provide further details of the 
wake formation at different particle positions relative to the trajectory.

Acknowledgements We thank Dr. M. Hyde of TSI Ltd. for his helpful assistance with the V3V system. This work was supported by Aquavitrum Ltd., the Leverhulme Trust and the Faculty of Engineering and the Environment of University of Southampton.

Data availability statement Pertinent data for this paper are available at https://doi.org/10.5258/SOTON/D0957.

Open Access This article is distributed under the terms of the Creative Commons Attribution 4.0 International License (http://creativeco mmons.org/licenses/by/4.0/), which permits unrestricted use, distribution, and reproduction in any medium, provided you give appropriate credit to the original author(s) and the source, provide a link to the Creative Commons license, and indicate if changes were made.

\section{References}

Andersen A, Pesavento U, Wang ZJ (2005a) Analysis of transitions between fluttering, tumbling and steady descent of falling cards. J Fluid Mech 541:91-104

Andersen A, Pesavento U, Wang ZJ (2005b) Unsteady aerodynamics of fluttering and tumbling plates. J Fluid Mech 541:65-90

Auguste F, Magnaudet J, Fabre D (2013) Falling styles of disks. J Fluid Mech 719:388-405

Belmonte A, Eisenberg H, Moses E (1998) From flutter to tumble: intertial drag and froude similarity in falling paper. Phys Rev Lett $81: 345-348$

Chrust M, Bouchet G, Dusek J (2013) Numerical simulation of the dynamics of freely falling discs. Physics of Fluids 25:044,102

Churst M, Bouchet G, J D (2013) Numerical simulation of the dynamics of freely falling discs. Physics of Fluids 25:044,102

Esteban LB, Shrimpton J, Ganapathisubramani B (2018) Edge effects on the fluttering characteristics of freely falling planar particles. Physical Review Fluids 3:064,302

Esteban LB, Shrimpton J, Ganapathisubramani B (2019) Study of the circularity effect on drag of disk-like particles. Int J Multiphase Flow 110:189-197

Feng J, Hu HH, Joseph DD (1994) Direct simulation of initial value problems for the motion of solid bodies in a newtonian fluid. part 1. sedimentation. J Fluid Mech 261:95-134

Fernandes P, Ern P, Risso F, Maugnadet J (2005) On the zigzag dynamics of freely moving axisymmetric bodies. Phys Fluids 17:098,107

Fernandes PC, Risso P, Ern P, Magnaudet J (2007) Oscillatory motion and wake instability of freely rising axisymmetic bodies. J Fluid Mech 573:479-502
Field SB, Klaus M, Moore MG, Nori F (1977) Chaotic dynamics of falling disks. Nature 388:252-254

Heisinger L, Newton P, Kanso E (2014) Coins falling in water. J Fluid Mech 714:243-253

Jayaweera KOLF (1972) An equivalent disc for calculating the terminal velocities of plate-like ice crystals. J Atmos Sci 29:596-597

Jones JM, Langton ARL, Ma L, M P, Williams A (2014) Pollutants generated by the combustion of solid biomass fuels. Springer

Kajikawa M (1992) Observations of the falling motion of plate-like crystals. part i: The free-fall patterns and velocity variations of unrimed crystals. J Meteor Soc Japan 70:1-9

Lee C, Su Z, Zhong H, Chen S, Zhou M, Wu J (2013) Experimental investigation of freely falling thin disks. part 2. transition of three-dimensional motion from zigzag to spiral. J Fluid Mech 42:77-104

List R, Schemenauer RS (1971) Free-fall behaviour of planar snow crystals, conical graupel and small hail. J Atmos Sci 28:110-115

Pothos S, Troolin D, Lai W, Menon R (2009) V3v- volumetric threecomponent velocitmetry for $3 \mathrm{~d}$ flow measurements - main principle, theory and applications. Termotehnica 2:25-32

Sabban L, van Hout R (2011) Measurements of pollen grain dispersal in still air and stationary near homogeneous, isotropic turbulence. J Aerosol Science 42:867-882

Smith EH (1971) Autorotating wings: an experimental investigation. J Fluid Mech 50:513-534

Tam D (2015) Flexibility increases lift for passive fluttering wings. J Fluid Mech 765:R2

Vincent L, Shambaugh WS, Kanso E (2016) Holes stabilize freely falling coins. J Fluid Mech 801:250-259

Willmarth WW, Hawk NE, Harvey RL (1964) Steady and unsteady motions and wakes of freely falling disks. Phys Fluids 7:197-208

Wilson L, Huang TC (1979) The influence of shape on the atmospheric settling velocity of volcanic ash particles. Earth Planet Sci Lett 44:311-324

Zhong H, Chen S, Lee C (2011) Experimental study of freely falling thin disks: transition from planar zigzag to spiral. Phys Fluids 23:11-702

Zhong H, Lee C, Su Z, Chen S, Zhou M, Wu J (2013) Experimental investigation of freely falling thin disks. part 1 . the flow structures and reynolds number effects on the zigzag motion. J Fluid Mech 716:228-250

Zhong HJ, Lee CB (2012) The wake of falling disks at low reynolds numbers. Acta Mech Sinica 28:367-371

Publisher's Note Springer Nature remains neutral with regard to jurisdictional claims in published maps and institutional affiliations.

\section{Affiliations}

\section{Luis Blay Esteban ${ }^{1}$ D . John Shrimpton ${ }^{1} \cdot$ Bharathram Ganapathisubramani $^{1}$}

Luis Blay Esteban lbe1g14@soton.ac.uk

John Shrimpton

John.Shrimpton@soton.ac.uk
Bharathram Ganapathisubramani

G.Bharath@soton.ac.uk

1 University of Southampton, Southampton, UK 